\title{
525.
}

\section{AN EXAMPLE OF THE HIGHER TRANSFORMATION OF A BINARY FORM.}

[From the Mathematische Annalen, vol. Iv. (1871), pp. 359-361.]

THE quartic

$$
(a, b, c, d, e)(x, y)^{4}
$$

is by means of the two quadrics

$$
(\alpha, \beta, \gamma)(x, y)^{2} \text { and }\left(\alpha^{\prime}, \beta^{\prime}, \gamma^{\prime}\right)(x, y)^{2}
$$

transformed into

(3)

$$
\left(a_{1}, b_{1}, c_{1}, d_{1}, e_{1}\right)\left(x_{1}, y_{1}\right)^{4}
$$

that is, eliminating $x, y$ from

$$
\begin{gathered}
(a, b, c, d, e)(x, y)^{4}=0, \\
x_{1}(\alpha, \beta, \gamma)(x, y)^{2}+y_{1}\left(\alpha^{\prime}, \beta^{\prime}, \gamma^{\prime}\right)(x, y)^{2}=0,
\end{gathered}
$$

we obtain

$$
\left(a_{1}, b_{1}, c_{1}, d_{1}, e_{1}\right)\left(x_{1}, y_{1}\right)^{4}=0
$$

It is required to express the invariants of (3) in terms of the simultaneous invariants of (1) and (2).

Write

$$
P, Q, R=\alpha x_{1}+\alpha^{\prime} y_{1}, \quad \beta x_{1}+\beta^{\prime} y_{1}, \quad \gamma x_{1}+\gamma^{\prime} y_{1}
$$

the equations from which $(x, y)$ have to be eliminated are

$$
(a, b, c, d, e)(x, y)^{4}=0, \quad(P, Q, R)(x, y)^{2}=0,
$$


and the result of the elimination therefore is

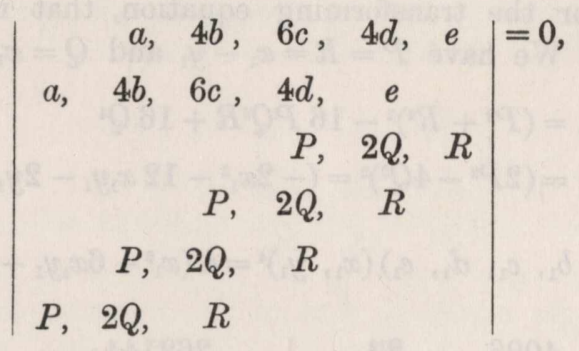

viz. this determinant is the transformed quartic $\left(a_{1}, b_{1}, c_{1}, d_{1}, e_{1}\right)\left(x_{1}, y_{1}\right)^{4}$.

The developed expression of the determinant is

$$
a^{2} R^{4}-8 a b Q R^{3}
$$

$$
\begin{aligned}
& +\left(\begin{array}{r}
-12 a c \\
+16 b^{2}
\end{array}\right) P R^{3}-24 a c Q^{2} R^{2}+\left(\begin{array}{r}
24 a d \\
-48 b c
\end{array}\right) P Q R^{2}-32 a d Q^{3} R \\
& +\left(\begin{array}{r}
2 a e \\
-32 b d \\
+36 c^{2}
\end{array}\right) P^{2} R^{2}+\left(\begin{array}{r}
-16 a e \\
+64 b d
\end{array}\right) P Q^{2} R+16 a e Q^{4} \\
& +\left(\begin{array}{r}
24 b e \\
-48 c d
\end{array}\right) P^{2} Q R-32 b e P Q^{3}+\left(\begin{array}{r}
-12 c e \\
+16 d^{2}
\end{array}\right) P^{3} R+24 c e P^{2} Q^{2} \\
& -8 d e P^{3} Q+e^{2} P^{4}
\end{aligned}
$$

so that writing for $P, Q, R$ their values, we have the transformed function $\left(a_{1}, b_{1}, c_{1}, d_{1}, e_{1}\right)\left(x_{1}, y_{1}\right)^{4}$, the coefficients being of the forms

$$
\begin{aligned}
& a_{1}=(a, b, c, d, e)^{2}(\alpha, \beta, \gamma)^{4} \\
& b_{1}=(\quad \quad \quad)^{2}(\alpha, \beta, \gamma)^{3}\left(\alpha^{\prime}, \beta^{\prime}, \gamma^{\prime}\right) \\
& \text { : } \\
& e_{1}=(\quad " \quad)^{2} . .\left(\alpha^{\prime}, \beta^{\prime}, \gamma^{\prime}\right)^{4} .
\end{aligned}
$$

Writing $I, J$ for the invariants of the quartic (1), and

$$
\begin{aligned}
& A=4\left(\alpha \beta^{\prime}-\alpha^{\prime} \beta\right)\left(\beta \gamma^{\prime}-\beta^{\prime} \gamma\right)-\left(\gamma \alpha^{\prime}-\gamma^{\prime} \alpha\right)^{2}, \\
& B=(e, c, a, b, c, d)\left(\alpha \beta^{\prime}-\alpha^{\prime} \beta, \gamma \alpha^{\prime}-\gamma^{\prime} \alpha, \beta \gamma^{\prime}-\beta^{\prime} \gamma\right)^{2},
\end{aligned}
$$

we have $I, J, A, B$ simultaneous invariants of the forms (1) and (2). Putting moreover $\nabla=I^{3}-27 J^{2}$, and writing $I_{1}, J_{1}, \nabla_{1}$, for the like invariants of the form (3), I find

$$
\begin{aligned}
& I_{1}=4\left(4 I B^{2}+12 J A B+\frac{1}{3} I^{2} A^{2}\right), \\
& J_{1}=8\left\{8 J B^{3}+\frac{4}{3} I^{2} A B^{2}+2 I J A^{2} B+\left(2 J^{2}-\frac{1}{27} I^{3}\right) A^{3}\right\},
\end{aligned}
$$

and thence

$$
\nabla_{1}=256\left(4 B^{3}-I A^{2} B-J A^{3}\right) \nabla
$$


As a verification, suppose $(a, b, c, d, e)(x, y)^{4}=x^{4}+y^{4}$ (whence $\left.I=1, J=0\right)$. And take $x_{1}(x+y)^{2}-y_{1}(x-y)^{2}=0$ for the transforming equation, that is, $(\alpha, \beta, \gamma)=(1,1,1)$ and $\left(\alpha^{\prime}, \beta^{\prime}, \gamma^{\prime}\right)=(-1,1,-1)$. We have $P=R=x_{1}-y_{1}$ and $Q=x_{1}+y_{1}$, and thence

that is,

$$
\begin{aligned}
\text { Det. } & =\left(P^{2}+R^{2}\right)^{2}-16 P Q^{2} R+16 Q^{4} \\
& =\left(2 P^{2}-4 Q^{2}\right)^{2}=\left(-2 x_{1}^{2}-12 x_{1} y_{1}-2 y_{1}^{2}\right)^{2},
\end{aligned}
$$

whence

$$
\left(a_{1}, b_{1}, c_{1}, d_{1}, e_{1}\right)\left(x_{1}, y_{1}\right)^{4}=4\left(x_{1}^{2}+6 x_{1} y_{1}+y_{1}^{2}\right)^{2}
$$

$$
I_{1}=\frac{4096}{3}, \quad=\frac{2^{12}}{3} ; \quad J_{1}=-\frac{262144}{27},=-\frac{2^{18}}{27}
$$

also

$$
A=-16, \quad B=8
$$

and the equations for $I_{1}, J_{1}$ become

$$
\begin{aligned}
\frac{4096}{3} & =4\left(4 \cdot 64+\frac{1}{3} 256\right) \\
-\frac{262144}{27} & =8\left(\frac{4}{3} \cdot-16 \cdot 64+\frac{1}{27} 4096\right),
\end{aligned}
$$

which are true. More generally, assuming

$$
(a, b, c, d, e)(x, y)^{4}=x^{4}+6 \Theta x^{2} y^{2}+y^{4},
$$

(whence $I=1+3 \Theta^{2}, J=\Theta-\Theta^{3}$ ), and the same transforming equation, we have whence

$$
\left(a_{1}, b_{1}, c_{1}, d_{1}, e_{1}\right)\left(x_{1}, y_{1}\right)^{4}=4\left\{(1+3 \Theta) x_{1}^{2}+(3-3 \Theta) 2 x_{1} y_{1}+(1+3 \Theta) y_{1}^{2}\right\}^{2},
$$

also

$$
I_{1}=\frac{2^{12}}{3}(1-3 \Theta)^{2}, \quad J_{1}=-\frac{2^{18}}{27}(1-3 \Theta)^{3}
$$

$$
A=-16, \quad B=8(1-\Theta) \text {. }
$$

Substituting these different values in the equations for $I_{1}, J_{1}$, we obtain

and

$$
16(1-3 \Theta)^{2}=12\left(1+3 \Theta^{2}\right)(1-\Theta)^{2}-72\left(\Theta-\Theta^{3}\right)(1-\Theta)+4\left(1+3 \Theta^{2}\right)^{2},
$$

$$
\begin{aligned}
-8(1-3 \Theta)^{3}= & 27\left(\Theta-\Theta^{3}\right)(1-\Theta)^{3}-9\left(1+3 \Theta^{2}\right)^{2}(1-\Theta)^{2} \\
& +27\left(1+3 \Theta^{2}\right)\left(\Theta-\Theta^{3}\right)(1-\Theta)-54\left(\Theta-\Theta^{3}\right)^{2}+\left(1+3 \Theta^{2}\right)^{3}
\end{aligned}
$$

which are in fact satisfied identically.

Cambridge, 26 July, 1871. 